\title{
Enhanced recovery after surgery (ERAS) decreases recovery time and improve the recovery effect versus for elective gastric cancer surgery: a single- center randomized controlled trial
}

\section{Shuhong Lv}

affiliated hospital of jiangsu university

\section{Xin Fan}

affiliated hospital of jiangsu university

Yan Li

affiliated hospital of jiangsu university

Jinling Yan

affiliated hospital of jiangsu university

Jing Xu

affiliated hospital of jiangsu university

qing wei ( 1140329223@qq.com)

affiliated hospital of jiangsu university https://orcid.org/0000-0002-5990-9513

\section{Research Article}

Keywords: enhanced recovery after surgery, postoperative recovery, pain intensity, postoperative ambulation, gastric cancer

Posted Date: May 14th, 2021

DOI: https://doi.org/10.21203/rs.3.rs-416991/v1

License: (c) (i) This work is licensed under a Creative Commons Attribution 4.0 International License. Read Full License 


\section{Abstract \\ Background}

Enhanced recovery after surgery (ERAS) can help patients improve recovery speed, improve patient satisfaction, reduce postoperative pressure and reduce postoperative hospital stay. This study evaluated ERAS feasibility and effectiveness compared with conventional perioperative care and their significance in gastrectomy for patients with gastric cancer.

\section{Methods}

This article is a prospective cohort analysis of 272 patients. This paper investigates tumor morphology, operation information, exhaustion time, ambulation time, walking distance on the operation day and 1 day, 2 days and 3 days after the operation, While blood test indexes related to postoperative recovery and postoperative complications are evaluated.

\section{Results}

The recovery of gastrointestinal function was faster in ERAS patients. There was a significant difference in the time of first exhaust between ERAS group and CC group ( $54.60 \pm 17.41 \mathrm{~h}$ vs. $76.71 \pm 9.47 \mathrm{~h} ; \mathrm{P<}$ 0.001). There were significant differences in the amount of diet on the day of operation, 1 day, 2 days and 3 days after operation between the two groups $(P<0.001)$; There was a significant difference in the time of first ambulation between eras group and CC group (1.14 $\pm 0.76 D$ vs. $2.74 \pm 0.98 D ; P<0.001)$. There were significant differences in walking distance between the two groups at operation day, 1 day, 2 days and 3 days after operation $(P<0.001)$. The postoperative hospital stay in the ERAS group was significantly shorter than that in the routine perioperative nursing group $(8.38 \pm 1.68 D, 11.18 \pm 1.38 D ; P<$ 0.001).

\section{Conclusion}

ERAS can reduce the stress after radical resection of gastric cancer, promote intestinal recovery, reduce pain intensity, and improve the recovery ability and satisfaction of patients.

\section{Introduction}

Gastric cancer is one of the most common malignant tumors in the world. According to recent estimates, 952000 new gastric cancer cases are diagnosed each year, making it the sixth most common malignant tumor globally. Furthermore, gastric cancer causes 732000 deaths a year, making it the fourth leading cause of cancer-related death. Over $40 \%$ of gastric cancer cases occur in China, and the incidence rate of gastric cancer is on the rise in recent years. 
Surgical treatment is still the only hope of cure. However, open resection is associated with severe trauma, numerous complications, slow recovery, prolonged hospital stay and many other perioperative complications. Laparoscopic radical gastrectomy is the first choice for the treatment of gastric cancer. For advanced gastric cancer, there is a high risk of recurrence and metastasis. Postoperative chemotherapy can improve the survival rate. Gastric cancer surgery is a complex operation with high risk related to postoperative stress, complications and sequelae. As poor postoperative recovery may delay the start of chemotherapy, surgeons pay more attention to methods to accelerate postoperative recovery.

The term enhanced recovery after surgery (ERAS) came into practice for the first time in 2002. Later, in 2009, emerging data from preliminary studies guided the issuance of the first consensus guidelines. Soon, principles first adopted in colorectal surgery became more commonly applied in other procedures. However, specific ERAS guidelines for gastric cancer were not published until 2014 (ERAS-GC). In China, the concept of ERAS was first introduced and promoted in 2007 by Li. In the same year, Li's team first reported the safety and effectiveness of ERAS in patients with gastric cancer.

Enhanced recovery after surgery (ERAS) is a multidisciplinary approach to perioperative care with evidence-supported measures to reduce surgical stress and accelerate postoperative rehabilitation. Typical ERAS protocols involve preoperative counselling, reduced fasting times, avoidance of bowel preparation, optimized anesthesia protocols, use of multimodal anesthesia, avoidance of nasogastric tubes and intraabdominal drains, early mobilization and early progression to food. ${ }^{6}$ ERAS has been applied to numerous surgical fields, including colorectal cancer surgery with success, including decreased lengths of stay, decreased costs, reduced surgical morbidity, and improved postoperative recovery.

International bodies have published various guidelines for the application of ERAS in different surgical fields, including colorectal surgery, pancreaticoduodenal surgery, weight-loss surgery, gynecology and breast surgery. Although ERAS core concept remains consistent, some of the measures differ with the type of surgery. Colorectal surgery has seen the earliest and most successful application of ERAS so far, with patients discharged 2-3 days after surgery. However, it remains to be seen if ERAS could be used for radical gastrectomy in the same way it has been applied with colorectal surgery. Many studies reported the application of ERAS in gastric cancer and achieved satisfactory results. However, most of the authors only studied the time before the first exhaust after ERAS, the first walking time to get out of bed and the time of hospitalization after ERAS, but did not study the walking distance of patients after gastric cancer operation. The walking distance is more valuable than the first time to walk out of bed. Walking distance can reflect patients' recovery state directly and continuously measure patients' walking distance, which is of better guidance for the study of patients' recovery state.

ERAS acts primarily by reducing the stress response during the perioperative period. The present study evaluates our ERAS protocols' feasibility and effectiveness in gastric cancer patients compared to conventional perioperative care undergoing gastrectomy. In addition to the first exhaust time, the first time out of bed and the first eating time studied by previous scholars, this study innovatively counted the 
distance of postoperative ambulation of patients, which provided a new clinical index for the analysis of postoperative recovery ability of patients with gastric cancer.

\section{Material And Methods}

\section{Ethics and consent}

This study was approved by the Ethics Committee of Affiliated Hospital of Jiangsu University. All procedures were in accordance with the ethical standards laid down in the 1964 Declaration of Helsinki and its later amendments. A written informed consent form was obtained from patients participating in this study.

\section{Patients}

Gastric cancer patients who underwent radical gastrectomy at the Affiliated Hospital of Jiangsu University between March 2018 and December 2019 were selected for this clinical trial. Clinical staging was determined according to the American Joint Committee on Cancer 7th edition - TNM (tumor, node, metastasis) staging of I-III for postoperative pathological diagnosis. The inclusion criteria were as follows:

1) diagnosis of gastric adenocarcinoma confirmed by a preoperative gastroscopy and pathological biopsy.

2) curative gastrectomy without simultaneous resection of other organs.

3) the age is between 18 and 80 .

4) non-emergency surgery.

5) no radiotherapy or chemotherapy before the operation.

6) the tumor has no long-term metastasis.

The ERAS group exclusion criteria were as follows:

a) radical resection could not be performed for any reason;

b) Judged by the doctor, it is not suitable for the participants.

c) Patients with ischemic heart disease, cerebrovascular disease, peripheral vascular disease, patients with cardiac function greater than 2 and those undergoing CABG operation, and patients with severe hypertension.

d) Patients with severe infection, respiratory insufficiency, coagulation dysfunction, severe liver and kidney dysfunction (child Pugh $\geq 10$ points; creatinine clearance rate less than $25 \mathrm{ml} / \mathrm{min}$ ); 
e) Opioids, non-steroidal drugs, cephalosporins and other commonly used drugs during hospitalization;

f) Have a history of gastric cancer surgery or complicated abdominal surgery

g) Gastric cancer complicated with bleeding, perforation, pyloric obstruction and other complications

h) Patients with cachexia and malnutrition (albumin less than or equal to $30 \mathrm{~g} / \mathrm{L}$, weight loss more than $10 \%$ in half a year, BMl less than $18, \mathrm{Hb}$ less than $70 \mathrm{~g} / \mathrm{L}$ )

i) Diabetic patients with metabolic complications

j) Patients who are unable to complete the accelerated rehabilitation surgery or have obvious contraindications.

k) Pregnant and lactating women.

Patients can also withdraw from the study after enrollment if any of the following conditions occur:

a) radical resection cannot be performed for any reason;

b) serious complications occur during or after surgery that requires rescue measures;

c) patients or their families request to withdraw from the study.

Data collection

All data were retrieved from the patients' clinical records. The items studied were the patients' characteristics, tumor profile, surgical information, postoperative data and postoperative complications. Patient characteristics evaluated include age, gender, body mass index (BMI), Nutritional Risk Screening (NRS) 2002 score and the risk grade of the American Society of Anesthesiologists (ASA) score. The pathological tumor stage (TNM classification) are also included. Surgical-related data included the kind of procedure and the type of reconstruction. Postoperative data included the time of the first flatus. The amount of diet on the operation day, the first day, the second day and the third day after operation were also collected. The time to get out of bed for the first time and the walking distance on the day of operation, the first day, the second day and the third day after the operation were gathered. Maximum pain on a visual analog scale (VAS, evaluated at operation day, the first, second and third day after surgery), postoperative hospital stays, postoperative complications are also collected. Blood samples were collected 1 day before surgery and the first day, the third day and the fifth day after surgery, the collected samples were used to analyze C-reactive protein (CRP) and Leukocyte Count (WBC) detection.

\section{ERAS program}

The ERAS program can be divided into preoperative, intraoperative and postoperative phases. Detailed information about the treatment and perioperative care was explained to the patients on admission. ${ }^{8}$, The 
ERAS protocol is shown in Table 1. 
Table 1

Enhanced recovery after surgery (ERAS) protocol.

\section{Time point Protocol}

On

admission

Day before

surgery

\section{Preoperative health education.}

2.No smoking or alcohol, prevention of cold.

3.Preoperative physical examination to assess nutritional status and rule out contraindications to surgery.

4.Chest breathing training: inhale deeply through the nose until respiratory exhaustion, hold the breath for one to two seconds ( this exercise can prolong the exchange time of oxygen and carbon dioxide in the lung, so that more oxygen enters the blood). Retract the lips, as by whistling, slowly exhale from the mouth and empty as you exhale. Six to eight times a day for 10 minutes. (take a rest after every 5 deep breaths) balloon blowing training to exercise your lung function is also applicable.

5.Teach correct and effective method of coughing and expectoration: before expectoration, cough gently for several times to loosen the sputum, then take a deep breath in the mouth, hold the breath, pause for a moment, cough once or twice with short force, discharge the sputum, and unfold your hands and press the abdomen, which can help you exert force and protect the wound after surgery.

6.Do physical exercise according to your physical strength every day.

7.If you suffer from severe malnutrition, i.e. weight loss $\geq 5 \%$ in one month, pleural effusion and ascites, or $70 \%-100 \%$ reduction in food intake (compared with the requirement) in the last week, we will give oral nutritional supplements. For those who can't eat, we will give preoperative intravenous drip of nutrient solution.

8.Nutritional risk screening and intervention: all patients with gastrointestinal cancer were screened with NRS (2002) nutritional risk screening table. For patients with a total score of $\geq 3$, oral enteral nutrition is recommended routinely; for patients with eating difficulties, parenteral nutrition solution can be used before and after the operation.

1.No routine bowel preparation (except in incidences of constipation).

2.Drink $600-1200 \mathrm{ml}$ of clear fluid 12 hours before the operation, such as Maltodextrin fructose.

3.Anesthesia consultation, skin preparation, blood examination, insertion of indwelling gastric tube and urinary catheter, and prophylactic antibiotics.

a The gastric tube could be removed when the amount of drainage was $\leq 100 \mathrm{~mL} / \mathrm{d}$; the drained fluid was not blood-tinged, and flatus had been passed.

b The abdominal drainage tube could be removed when abdominal infection, anastomotic fistula, and other postoperative complications were ruled out, and the drainage volume was $\leq 10 \mathrm{~mL} / \mathrm{d}$ for 2 days. 


\section{Time point Protocol}

Day of

surgery
1.Drink $200 \mathrm{ml}$ Carbohydrate electrolyte solution 2 hours before operation; responsible ward nurse will communicate with the operating room to determine the operation time and clear liquid time as far as possible.

2.Electric blanket and abdominal-temperature saline irrigation to maintain body temperature during surgery.

3.Decision to use abdominal drainage tubes depended on the surgical conditions of antibiotics.

4.Subcutaneous infiltration anesthesia + intravenous/epidural analgesic pump anesthesia + intravenous nonsteroidal anti-inflammatory drug (e.g., $40 \mathrm{mg}$ parecoxib sodium (Pharmacia and Upjohn Company, USA)) + 5 mg Dexamethasone sodium phosphate injection.

5.Routine prevention of nausea and vomiting for 2-3 days.

6.Attempt to drink warm water (about $20 \mathrm{~mL} / \mathrm{h}$ ) after the patient revived.

Postoperative day 1
1.After operation, water can be taken orally under the guidance of medical staff, and the amount of water will gradually increase according to the recovery of intestinal peristalsis and tolerance. According to the doctor's advice: after the recovery of general bowel sounds liquid diet can commence, anal exhaust after surgery into a semi-liquid diet, high protein, high vitamin, low fat and low sugar diet is recommended for patients who could not meet the nutritional needs after oral administration were given intravenous nutrition supplement.

2.Early mobilization encouraged and establish activity diary. On the first day after the operation, bed activity should be 1-2 hours, divided into 10-15 minutes, 50-100 meters each time. After that, they should get out of bed for 4-6 hours every day, 1000-2000 meters per day. After the operation, the hip and ankle pump, limb massage and turning over cough and expectoration were started as soon as possible.

\section{Gastric tube removed according to accepted criteria for extubation ${ }^{\text {a }}$}

4.Intermittent urinary catheterization started to train the bladder and stopped when bladder sensation returned to normal. Catheter tube should be removed according to accepted criteria for extubation.

5. If necessary, analgesics were injected twice a day within 3 days after operation. (e.g., $40 \mathrm{mg}$ parecoxib sodium (Pharmacia and Upjohn Company, USA) + $5 \mathrm{mg}$ Dexamethasone sodium phosphate injection).

a The gastric tube could be removed when the amount of drainage was $\leq 100 \mathrm{~mL} / \mathrm{d}$; the drained fluid was not blood-tinged, and flatus had been passed.

b The abdominal drainage tube could be removed when abdominal infection, anastomotic fistula, and other postoperative complications were ruled out, and the drainage volume was $\leq 10 \mathrm{~mL} / \mathrm{d}$ for 2 days. 


\section{Time point Protocol}

Postoperative 1.Encouragement to continue and prolong out-of-bed activities.

day $2-7$

2.Oral fluid intake needs to be increased, liquid diet (such as small amounts of rice soup) started, intravenous fluids reduced.

3.Antibiotics stopped if there was no evidence of infection.

4.Gastric tube removed if not obstructed. ${ }^{\text {a }}$

5.If urinary catheter was not obstructed, ${ }^{b}$ it was removed after completion of bladder training.

a The gastric tube could be removed when the amount of drainage was $\leq 100 \mathrm{~mL} / \mathrm{d}$; the drained fluid was not blood-tinged, and flatus had been passed.

${ }^{\mathrm{b}}$ The abdominal drainage tube could be removed when abdominal infection, anastomotic fistula, and other postoperative complications were ruled out, and the drainage volume was $\leq 10 \mathrm{~mL} / \mathrm{d}$ for 2 days.

Conventional perioperative care (Traditional program)

Patients in the conventional surgery group received conventional perioperative care: 1) f fasting for 10 hours before the operation, stopping fluid intake 6 hours before operation; 2) bowel preparation (enemas and oral antibiotics), 3) nasogastric tube and peritoneal drainage tube placement, 4) administration of general anesthesia, 5) resumption of diet after the first flatus, and 6) resumption of ambulation 2-3 days after surgery.

Patients in the traditional perioperative care group were allowed to eat a liquid diet until lunch of the day before surgery and no food was allowed after dinner. Endotracheal intubation was performed under general anesthesia. Anesthesia was general anesthesia without a combination of local preemptive analgesia. Postoperative pain was managed by PCA only. NSAIDs were not routinely used in the CC group. Additional painkillers were not routinely given and additional analgesics were administered only when the patient complained about pain. A nasogastric tube was used for stomach decompression before surgery and removed after the bowel function recovered completely.

Postoperative treatment consisted of parenteral nutrition of fat emulsion, amino acids and glucose (11\%) injection, which was administered until flatus. At that time, the nasogastric tube was removed and the patients were advised to drink water. After full intestinal recovery, the diet consisted of a clear liquid diet, then a full liquid diet, and finally a soft diet. In the CC group, the urinary catheters were removed 2-3 days after surgery. Drains routinely were used and the drainage tubes were removed 5 or 6 days after operation if the drainage fluid was clear and the amount of drainage discharge was less than $100 \mathrm{~mL} /$ day. Ambulation was encouraged 24 hours after surgery in this group and all patients were mobilized on the second day of surgery in the CC group.

Discharge criteria 
Patients were discharged based on the following criteria: 1) normal body temperature; 2) adequate pain control with oral medication; 3 ) absence of nausea and/or vomiting; 4) good flatus and/or defecation; 5) ability to tolerate non-elemental diet and soft food without intravenous nutritional support; 6) mobilization without assistance; 7) normal laboratory data; 8) no postoperative complications; and 9) acceptance of discharge by the patient.

Follow-up

In the ERAS group, the patients kept in touch with us by an outpatient service or telephone after discharge within the first 24 hours and once weekly for 4 weeks. The patients could also contact us if they had any discomfort. Readmission was considered if any of the following occurred: hyperpyrexia, abdominal pain, bowel obstruction, gastrointestinal hemorrhage, infection or poor healing of the wound.

\section{Statistical analysis}

Comparisons between the study groups were performed using Student's t-test, Chi-squared test, MannWhitney $\mathrm{U}$ test, or Fisher's exact test as appropriate. Continuous variables are represented as the mean \pm standard deviation (SD) and were analyzed using Student's t-test or Mann-Whitney U-test. Categorical variables are presented as counts and percentages and were analyzed using the Chi-squared test or the Fisher's exact test. All statistical tests were two-tailed, and a value of $\mathrm{P}<0.05$ was considered statistically significant. All statistical analyses were performed using SPSS 26.0 statistics software (SPSS Inc., Chicago, IL, USA).

Patient characteristics

A total of 272 patients were included in the study, 175 females and 97 males, with a mean age of 62.87 years $\left(62.87 \pm 8.73\right.$ years) and a mean body mass index of $22.77 \mathrm{~kg} / \mathrm{m}^{2}$ (range: $\left.15.04-32.03 \mathrm{~kg} / \mathrm{m}^{2}\right)$ (Table 2).

After statistical analysis, we found that there were no significant differences between the ERAS group and the $C C$ group in terms of age $(P=0.338)$, gender $(P=0.574)$, BMI $(P=0.298)$, NRS 2002 score $(P=0.144)$, ASA score $(P=0.943)$ and TNM classification $(P=0.164)$ (Table 2$)$.

There were 145 patients (53.3\%) in NRS 2002 score 1 and 127 patients (46.7\%) in NRS 2002 score 2. There were 148 patients (54.4\%) in ASA class I and 124 patients (45.6\%) in ASA class $\mathbb{~}$.

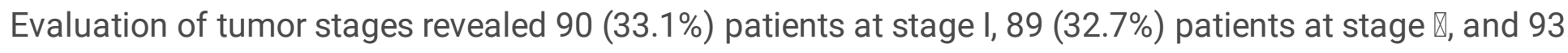
$(34.2 \%)$ patients at stage $\llbracket$.

Surgical procedures and outcomes

Surgical approach 
There was no statistical significance in the surgical approach between the ERAS and CC groups $(P=$ 0.136) (Table 3). The percentage of Laparoscopic-assisted in the ERAS group was $73.33 \%$, and $80.92 \%$ in the CC group.

Type of operation

There was no statistical significance in the type of operation between the ERAS and CC groups $(P=$ 0.190 ) (Table 3). The percentage of Total gastrectomy in the ERAS group was $83.33 \%$, and $88.81 \%$ in the CC group. 
Table 2

Comparison of clinicopathologic characteristics between the ERAS and CC groups

\begin{tabular}{|c|c|c|c|}
\hline Characteristics & ERAS group & CC group & $P$-value \\
\hline Age & & & 0.338 \\
\hline$\leq 62$ & 47 & 51 & \\
\hline$>62$ & 73 & 101 & \\
\hline Mean & 62.15 & 63.44 & \\
\hline Median & 62 & 64 & \\
\hline Gender & & & 0.574 \\
\hline Male & 45 & 52 & \\
\hline Female & 75 & 100 & \\
\hline BMI (kg/m2) & $22.56 \pm 2.60$ & $22.94 \pm 3.17$ & 0.298 \\
\hline NRS 2002 score & & & 0.144 \\
\hline 1 & 58 & 87 & \\
\hline 2 & 62 & 65 & \\
\hline ASA score & & & 0.943 \\
\hline I & 65 & 83 & \\
\hline II & 55 & 69 & \\
\hline TNM classification & & & 0.164 \\
\hline I & 44 & 46 & \\
\hline II & 32 & 57 & \\
\hline III & 44 & 49 & \\
\hline
\end{tabular}


Table 3

Comparison of surgical procedures and outcomes between the ERAS and CC groups.

\begin{tabular}{|llll|}
\hline Characteristics & ERAS group & CC group & $P$-value \\
\hline Surgical approach & & & 0.136 \\
\hline Laparoscopic assisted & 88 & 123 & \\
Open & 32 & 29 & 0.190 \\
\hline Type of operation & & & \\
\hline Distal gastrectomy & 20 & 17 & \\
\hline Total gastrectomy & 100 & 135 & \\
\hline Abbreviations: ERAS, enhanced recovery after surgery; CC, conventional care. &
\end{tabular}

Comparison of postoperative recovery

Gastrointestinal function recovery

Compared with the CC group, the patients in the ERAS group showed significantly accelerated recovery of gastrointestinal function in terms of time to first flatus $(P<0.001)$ (Table 4$)$. The mean time to first flatus was $54.60 \pm 17.41$ postoperative hours in the ERAS group and $76.71 \pm 9.47$ postoperative hours in the CC group.

It can be seen from Table 4 that the daily food intake of the ERAS group and CC group on the day of operation, first day after operation, second day after operation and third day after operation are statistically different $(P<0.001)$. CC group did not have food on the day of operation, first day and second day after operation, while in the ERAS group, some patients could have food on the day of operation. Through Table 4, we can indicate that the daily food intake of patients in the ERAS group was increasing within the four days after operation.

There was also a significant difference in the time of first ambulation after operation between ERAS group and CC group $(P<0.001)$. The average first time out of bed time was $1.14 \pm 0.76$ days in the ERAS group and $2.74 \pm 0.98$ days in the CC group. At the same time, the walking distance of patients out of bed was statistically different. In the ERAS group, some patients could get out of bed and walk $3.48 \pm 14.15$ meters on average, while no patients in the CC group could get out of bed on the day of operation. On the first day after operation, the walking distance of the ERAS group and CC group was $81.61 \pm 78.45$ vs 1.25 \pm 3.51 meters $(P<0.001)$. On the second day, the walking distance between the ERAS group and CC group was $200.01 \pm 182.94$ vs $5.13 \pm 7.76$ meters $(P<0.001)$. On the third day after operation, the walking distance between the ERAS group and the CC group was $307.68 \pm 217.19$ vs $27.37 \pm 19.01$ meters $(P<$ 0.001). The activity of the ERAS group was better than that of the CC group.

Postoperative hospital stays 
Postoperative hospital stays were significantly shorter in patients receiving ERAS treatment than in those patients who received conventional perioperative care $8.38 \pm 1.68$ vs $11.18 \pm 1.38$ days; $P<0.001$ )

(Table 4).

Table 4

Comparison of postoperative clinical outcomes between ERAS and CC groups

\begin{tabular}{|llll|}
\hline Characteristics & ERAS group & CC group & $P$-value \\
\hline Time to first flatus $(\mathrm{h})$ & $54.60 \pm 17.41$ & $76.71 \pm 9.47$ & $<0.001$ \\
\hline Food intake on Operation day $(\mathrm{ml})$ & $71.91 \pm 71.60$ & $0 \pm 0$ & $<0.001$ \\
\hline Food intake on the first day after operation $(\mathrm{ml})$ & $308.58 \pm 188.72$ & $0 \pm 0$ & $<0.001$ \\
\hline Food intake on the second after operation $(\mathrm{ml})$ & $356.98 \pm 205.87$ & $0 \pm 0$ & $<0.001$ \\
\hline Food intake on the third day after operation(ml) & $413.79 \pm 197.99$ & $16.18 \pm 9.27$ & $<0.001$ \\
\hline First time out of bed(d) & $1.14 \pm 0.76$ & $2.74 \pm 0.98$ & $<0.001$ \\
\hline Activity distance on the Operation day(metre) & $3.48 \pm 14.15$ & $0 \pm 0$ & $=0.003$ \\
\hline Activity distance on the first day after operation(metre) & $81.61 \pm 78.45$ & $1.25 \pm 3.51$ & $<0.001$ \\
\hline Activity distance of the next day after operation(metre) & $200.01 \pm 182.94$ & $5.13 \pm 7.76$ & $<0.001$ \\
\hline $\begin{array}{l}\text { Activity distance of the third day after } \\
\text { operation(metre) }\end{array}$ & $307.68 \pm 217.19$ & $27.37 \pm 19.01$ & $<0.001$ \\
\hline Postoperative hospital stays(days) & $8.38 \pm 1.68$ & $11.18 \pm 1.38$ & $<0.001$ \\
\hline Abbreviations: ERAS, enhanced recovery after surgery; CC, conventional care; & \\
\hline
\end{tabular}

Pain control

VAS analysis showed that pain intensity of patients in the ERAS group was significantly lower than that of patients in the CC group on 1 hour $(P<0.001), 1$ day $(P<0.001), 2$ days $(P<0.001)$ and 3 days $(P<$ 0.001 ) after operation(Table 5).

Table 5

Comparison of VAS between the ERAS group and CC groups

\begin{tabular}{|llll|}
\hline Characteristics & ERAS group & CC group & $P$-value \\
\hline VAS 1 hour after operation & $2.50 \pm 0.65$ & $3.44 \pm 0.93$ & $<0.001$ \\
\hline VAS 1 day after operation & $2.53 \pm 1.05$ & $4.00 \pm 0.76$ & $<0.001$ \\
\hline VAS 2 days after operation & $1.96 \pm 0.72$ & $3.03 \pm 0.61$ & $<0.001$ \\
\hline VAS 3 days after operation & $1.35 \pm 0.48$ & $2.58 \pm 0.50$ & $<0.001$ \\
\hline Abbreviations: ERAS, enhanced recovery after surgery; CC, conventional care. \\
\hline
\end{tabular}

Postoperative surgical stress and inflammatory response 
The response induced by systemic surgical stress was assessed by measuring the white blood cell (WBC) count and C-reactive protein levels. When comparing the WBC count before surgery between the groups, there was no statistical significance $(P=0.564)$ (Table 6). The WBC count in both groups was elevated on the first day after operation, but compared to the ERAS group on the first day after operation, the WBC in the $C C$ group was significantly higher $(P=0.001)$. The white blood cell $(W B C)$ count on the first day after operation is $12.51 \pm 3.34$ in the ERAS group and $14.45 \pm 1.81$ in the CC group. The WBC count in the ERAS group and $\mathrm{CC}$ group both began to drop on the third day after operation, but there are also statistical significances on the third day $(P=0.023)$ and fifth day $(P=0.001)$ after operation between the ERAS group and CC group.

For the inflammatory marker $\mathrm{C}$-reactive protein, there was no statistical significance between the ERAS and CC groups before surgery $(P=0.280)$ (Table 4$)$. There was statistical significance for $C$-reactive protein on the first day, third day and fifth day after operation between the two groups $(P<0.01)$ (Table 4$)$. The median CRP (range) level decreased from $53.23 \pm 16.51 \mathrm{mg} / \mathrm{dL}$ to $50.73 \pm 17.87 \mathrm{mg} / \mathrm{dL}$ on the third day after surgery and drop to $22.55 \pm 12.41 \mathrm{mg} / \mathrm{dL}$ on the fifth day after surgery in the ERAS group and from $60.62 \pm 20.27 \mathrm{mg} / \mathrm{dL}$ to $56.67 \pm 23.82 \mathrm{mg} / \mathrm{dL}$ at the third day three after surgery and drop to $26.97 \pm$ $9.79 \mathrm{mg} / \mathrm{dL}$ at the fifth day after surgery in CC group. Furthermore, compared with the CC group, the level of CRP in the ERAS group was also lower on the first day $(P<0.001)$, third day $(P<0.001)$ and fifth day ( $P$ $<0.001)$ after operation.

Table 6

Comparison of laboratory findings between the ERAS and CC groups

\begin{tabular}{|llll|}
\hline Characteristics & ERAS group & CC group & $P$-value \\
\hline White blood cell count $\left(10^{\wedge} 9\right)$ & & & \\
\hline Before surgery & $5.92 \pm 1.27$ & $6.19 \pm 2.43$ & $=0.564$ \\
\hline first day after operation & $12.51 \pm 3.34$ & $14.45 \pm 1.81$ & $=0.001$ \\
\hline third day after operation & $8.86 \pm 2.79$ & $10.75 \pm 1.58$ & $=0.024$ \\
\hline fifth day after operation & $6.68 \pm 2.14$ & $7.88 \pm 1.52$ & $=0.001$ \\
\hline CRP (mg/dL) & & & \\
\hline Before surgery & $4.52 \pm 1.23$ & $4.61 \pm 1.34$ & $=0.280$ \\
\hline first day after operation & $53.23 \pm 16.51$ & $60.62 \pm 20.27$ & $<0.001$ \\
\hline third day after operation & $50.73 \pm 17.87$ & $56.67 \pm 23.82$ & $<0.001$ \\
\hline fifth day after operation & $22.55 \pm 12.41$ & $26.97 \pm 9.79$ & $<0.001$ \\
\hline Abbreviations: ERAS, enhanced recovery after surgery; CC, conventional care; CRP, C-reactive protein; \\
\hline
\end{tabular}

Postoperative complications 
No statistical significance was found between the incidences of postoperative complications in the ERAS and CC groups $(P=0.943)$ (Supplementary Table 1$)$. A total of $17(14.17 \%)$ patients experienced postoperative complications in the ERAS group and 22 (14.47\%) patients in the CC group developed postoperative complications (Supplementary table1). The operative complications were anastomotic leakage (ERAS 2, CC $3 ; P=0.943$ ), intra-abdominal infection (ERAS 2, CC 4; $P=0.852$ ), surgical incision infection (ERAS 3, CC 4; $P=0.946$ ), incision fat liquefaction (ERAS 4, CC 5; P = 0.984), and gastroparesis (ERAS 6, CC 6; $P=0.675$ ).

\section{Discussion}

In the present study, we demonstrated the effectiveness and feasibility of our ERAS program in gastric cancer patients who underwent radical gastrectomy by open surgery or laparoscopy. Compared with the conventional perioperative care group, the ERAS group had a faster first flatus $(P<0.001)$, faster first ambulation $(P<0.001)$, further walking distance $(P<0.001)$ after operation, patients experienced lesser pain $(P<0.001)$ and had a shorter postoperative $(P<0.001)$. These findings are consistent with those of previous studies.

Wilmore and Kehlet first reported the ERAS programs, which apply a series of optimized perioperative measures with evidence-based medicine to reduce surgical trauma and stress, to accelerate postoperative rehabilitation, and to shorten the length of stay. ${ }^{26}$, ERAS was first used after colorectal cancer surgery, and then, ERAS protocols have been gradually accepted as being able to optimize clinical outcomes, value and experience for patients with GC. ${ }^{8}$ Subsequently, certain gastric cancer treatment centers have applied ERAS programs to gastrectomy' that confirms their safety and effectiveness.

Expediting postoperative bowel function and advancing of diet are important targets of ERAS protocols and were successfully achieved in GC-ERAS group. In a similar Japanese study with an HC group, ${ }^{35}$ the first flatus occurred one day earlier and the first bowel movement occurred two days earlier in the GCERAS group. This result was further verified in a randomized prospective study by Wang et al., ${ }^{34}$ who reported that flatus occurred one day earlier in patients on an ERAS protocol.

Early oral feeding was safe. ${ }^{21} 17,21$ Early postoperative enteral nutrition with dietary fiber could accelerate recovery of peristalsis, protect gut mucosal barrier function, alleviate intestinal barrier dysfunction, decrease the incidence of bacterial translocation and enhance the recovery of gut function.24 In the present study, most patients who underwent ERAS tolerated early oral diet well. Although gastroparesis occurred in some patients, the symptoms mostly occurred in the initial stage of oral diet and did not develop into severe complications $(P=0.675)$. Several studies showed that ERAS resulted in significantly reduced postoperative hospital stays for gastrectomy. ${ }^{34} 7 \mathrm{It}$ was reported that the postoperative hospital stay in gastric cancer patients could be decreased to 3.8 days in the ERAS group. 25 In the present study, ERAS patients had a mean postoperative hospital stay of $8.38 \pm 1.68$ days and $11.18 \pm 1.38$ days in the CC group. The results of our study suggested that postoperative recovery was significantly enhanced by our ERAS protocol in gastric cancer patients undergoing gastrectomy. 
Preoperative oral administration of carbohydrates, which is extensively recognized as an important component of ERAS programs, accelerates the early release of insulin and avoids postoperative insulin resistance and excessive protein degradation. However, preoperative oral carbohydrates do not improve postoperative nutritional status or contribute to maintaining muscle strength. ${ }^{21}$

The time to initial liquid diet intake, out-of-bed activity period and time to postoperative gastrointestinal function recovery were significantly shorter than those in the control group under the premise of the same recovery effect.

The ERAS concept emphasizes that patients get out of bed, ingest a liquid diet, and undergo removal of the gastrointestinal decompression tube and catheter earlier to promote postoperative intestinal function recovery and accelerated rehabilitation.

While surgical resection is still the only option to cure gastric cancer, it can lead to a strong stress response. Stress inhibits immune function and stimulates the production of several inflammatory mediators, which further inhibit immune function. These changes result in increased postoperative complications, delayed recovery after surgery, and other adverse effects. ${ }^{23}$ The core mechanism of ERAS is that multimodal interventions may lead to a major reduction in the undesirable sequelae of surgical injury, and stress-free surgery is the key goal of ERAS. Robust evidence suggested that ERAS played an important role in attenuating the surgical stress response and accelerating the return to baseline in colorectal cancer surgery ${ }^{18}$, which was afforded eloquent proof in GC surgery. The inflammatory factors, such as CRP, IL- 6 and tumor necrosis factor a, are related to the extent of tissue injury caused by surgery.

CRP is an inflammatory protein synthesized and secreted by the liver in the acute phase of stress. It can activate complement, promote granulocyte proliferation, and enhance macrophage phagocytosis. Postoperative elevation in serum CRP level is closely related to the degree of surgical trauma. CRP can reflect acute stress and inflammatory response with high sensitivity and specificity. Some researchers have reported that CRP is an independent predictor of prognosis in gastric cancer patients. ${ }^{30}$ In our study, the difference in CRP level between the two groups suggest that the application of ERAS reduced the inflammatory response in gastric cancer patients.

C-reactive protein (CRP), an important systemic inflammatory biomarker, is extensively used in clinical settings for infection diagnosis.' This is in accordance with the clinical experience that abnormal CRP level is very common in the early days after surgery. Considering a possible physiological fluctuation by transient bacterial contamination during the operation or preparation of intestinal anastomosis. The authors concluded that such results indicated that CRP on POD3 could be used for early oral diet advancement within an ERAS program.' The reason for the remarkably enhanced diagnostic capacity of CRP may be that, on the fifth day after surgery, the mixing effects such as surgical trauma, blood loss, or absorption of necrotic tissues that are known to increase CRP levels were usually well controlled. 
Garcia-Granero et al. published a study with 250 patients after colorectal operation and likewise found that CRP on POD 5 was an eligible marker for early discharge, as its specificity was $83 \%$ and NPV was $98 \% .26$ Benoit et al. suggested that a CRP before POD 5 of $<100 \mathrm{mg} / \mathrm{L}$ was reassuring and may permit the patient to leave the hospital in safe conditions.'

ERAS aims to improve outcomes and promote early discharge by emphasizing preoperative patient education, shortening the duration of preoperative fasting, supplying preoperative carbohydrates, no bowel preparation, active prevention of hypothermia, no routine use of nasogastric tubes, controlling pain sufficiently without opioids, providing early ambulation, and quickly advancing the return to a normal diet. Studies have found that some procedures in gastrectomy, for example, routine nasogastric decompression were unnecessary because early oral feeding enhances the postoperative gastrointestinal tract recovery and decreased the duration of hospital stay without increasing complications.

ERAS encompassed a combination of preoperative, intraoperative and postoperative measures to enhance the postoperative recovery in surgical procedures. Compared with conventional care, ERAS could reduce the stress response and organ dysfunction, shorten the duration to flatus and defecation, accelerate the decrease in CRP and WBC, and thereby greatly shorten the postoperative stay, fasten the recovery of gut function, shorten the time required for overall recovery and increase patients' satisfaction. ${ }^{3{ }^{\prime \prime}}$ Our results were consistent with the findings of other studies. ${ }^{36}$ We found that in our study, ERAS was able to reduce postoperative stress and inflammation. In the present study, we found that patients after gastrectomy in the ERAS group had a shorter hospital stay $(P<0.001)$ and postoperative complications had no statistical significance $(P=0.943)$.

Preoperative patient education through contact between patients and staff can avoid the anxiety and perioperative stress reactions, which promotes faster recovery. Optimal pain control plays a fundamental role in postoperative care. Postoperative pain is one of the most important factors that delays postoperative recovery by not only increasing surgical stress but also affecting the mobilization of patients.' The results showed that pain intensity in the ERAS group was significantly lower than that of the $C C$ group $(P<0.001)$. Wang et al7 reported that the first day of flatus after gastrectomy was faster in patients who received ERAS care than in those who received conventional care. Our result is similar to his findings $(P<0.001)$, times to first flatus in the ERAS group were $54.60 \pm 17.41$ hours and $76.71 \pm 9.47$ hours in the $\mathrm{CC}$ group. Prolonged perioperative fasting, preoperative bowel preparation, and nasogastric tube intubation were likely to delay bowel-function recovery. Previous studies have shown that the small intestine might return to normal enteroinesia 6 hours after abdominal surgery and that liquid can be easily absorbed in the small intestine in early postoperative recovery.

\section{Conclusion}

ERAS can reduce postoperative stress, enhance the recovery of the gut, reduce pain intensity, shorten hospital stays and increase satisfaction in gastric cancer patients undergoing curative gastrectomy. 


\section{Declarations}

Acknowledgements

The authors acknowledge all the volunteer donors involved in this study. We would like to thank Shuhong Lv and his entire ERAS team in the general surgery unit of Affiliated Hospital of Jiangsu University for all the support they have provided upon completion of our paper.

Consent of publication

Not applicable

Availability of data and materials

The datasets used and/or analyzed during the present study are available from the corresponding author on reasonable request.

Funding

This research did not receive any specific grant from funding agencies in the public, commercial, or notfor-profit sectors. It is supported by The Foundation for Young Scientists of Affiliated Hospital of Jiangsu University (Grant numbers: JDFYRC2016002) and Jiangsu Provincial Medical Youth Talent (Grant numbers: QNRCQ2016839) and Zhenjiang Science and Technology Pillar Program (Grant numbers: SH2018082) and Zhenjiang Science and Technology Innovation Fund (Grant numbers: RK2020034).

Authors' contributions

SL and QW designed the study. YL acquired the data. LS and XF wrote the manuscript. JY and JX analyzed the results. SL and QW edited the manuscript. All authors read and approved the final manuscript.

Ethics approval and consent to participate

The experimental protocol used in the present study was approved by the Ethics Committee for Human Subject study of Jiangsu University (Zhenjiang, China). All patients provided written informed consent.

Patient consent for publication

Not applicable.

Disclosure of conflict of interest

None.

\section{References}


[1] Siegel RL, Miller KD, Jemal A. Cancer Statistics, 2017. CA Cancer J Clin 2017; 67: 7-30, doi: 10.3322/caac.21387.

[2] Ferro A, Peleteiro B, Malvezzi M, Bosetti C, Bertuccio P,Levi F, et al. Worldwide trends in gastric cancer mortality (1980-2011), with predictions to 2015, and incidence by subtype. Eur J Cancer 2014; 50: 13301344, doi: 10.1016/j.ejca.2014.01.029.

[3] Li G, Hu Y, Liu H. Current status of randomized controlled trials for laparoscopic gastric surgery for gastric cancer in China. Asian J Endosc Surg 2015; 8: 263-267, doi: 10.1111/ases.12198.

[4] Tegels JJ, De Maat MF, Hulsewe KW, Hoofwijk AG, Stoot JH. Improving the outcomes in gastric cancer surgery. World J Gastroenterol 2014; 20: 13692-13704, doi: 10.3748/w

[5] Son T, Hyung WJ. Laparoscopic gastric cancer surgery:Current evidence and future perspectives. World J Gastroenterol 2016; 22: 727-735, doi: 10.3748/wjg.v22.i2.727.

[6] Lassen K, Soop M, Nygren J, et al. Consensus review of optimal perioperative care in colorectal surgery: Enhanced Recovery After Surgery (ERAS) Group recommendations. Arch Surg 2009;144:961-9.

[7] Mortensen K, Nilsson M, Slim K, et al. Consensus guidelines for enhanced recovery after gastrectomy: Enhanced Recovery After Surgery (ERAS(R)) Society recommendations. Br J Surg 2014;101:1209-29.

[8] Jiang Z, Li J. Current Status of Enhanced Recovery After Surgery in China. Zhonghua Wei Chang Wai Ke Za Zhi 2016;19:246-9.

[9] K.Mortensen, M.Nilsson, K.Slim, M.Sch€afer, C.Mariette, M.Braga, F.Carli, N.Demartines, S.M.Griffin, K.Lassen, Consensus guidelines for enhanced recovery after gastrectomy, Br.J.Surg.101 (2014)12091229, https://doi.org/10.1002/bjs. 9582.

[10] K.K.Varadhan, K.R.Neal, C.H.C.Dejong, K.C.H.Fearon, O.Ljungqvist, D.N.Lobo, The enhanced recovery after surgery (ERAS) pathway for patients undergoing major elective open colorectal surgery: a metaanalysis of randomized controlled trials, Clin. Nutr. 29 (2010) 434-440, https://doi.org/10.1016/j.clnu.2010.01.004.

[11] Pedziwiatr M, Kisialeuski M, Wierdak M, Stanek M, Natkaniec M, Matlok M, et al. Early implementation of Enhanced Recovery After Surgery (ERAS(R)) protocol - Compliance improves outcomes: A prospective cohort study. Int J Surg 2015; 21: 75-81, doi: 10.1016/j.ijsu.2015.06.087.

[12] Lassen K, Coolsen MM, Slim K, Carli F, de Aguilar-Nascimento JE, Schafer M, et al. Guidelines for perioperative care for pancreaticoduodenectomy: Enhanced Recovery After Surgery (ERAS(R)) Society recommendations. Clin Nutr 2012; 31: 817-830, doi: 10.1016/j.clnu.2012.08.011.

[13] Clinical Outcomes of Surgical Therapy Study Group, Nelson H, Sargent DJ, et al. A comparison of laparoscopically assisted and open colectomy for colon cancer. N Engl J Med 2004;350:2050-9. 
[14] Carli F. Physiologic considerations of Enhanced Recovery After Surgery (ERAS) programs: implications of the stress response. Can J Anaesth 2015; 62: 110-119, doi: 10.1007/s12630-014-0264-0.

[15] Ljungqvist O, Scott M, Fearon KC. Enhanced recovery after surgery: a review. JAMA Surg. 2017;152(3):292.

[16] Makuuchi R, Sugisawa N, Kaji S, Hikage M, Tokunaga M, Tanizawa Y, et al. Enhanced recovery after surgery for gastric cancer and an assessment of preoperative carbohydrate loading. Eur $\mathrm{J}$ Surg Oncol 2017; 43: 210-217, doi: 10.1016/j.ejso.2016.07.140.

[17] Wilmore DW, Kehlet H. Management of patients in fast track surgery. BMJ 2001;322:473-6. doi: 10.1136/bmj.322.7284.473.

[18] Kehlet H, Wilmore DW. Evidence-based surgical care and the evolution of fast-track surgery. Ann Surg 2008;248:189-98. doi: 10.1097/SLA.0b013e31817f2c1a.

[19] Mingjie X, Luyao Z, Ze T, YinQuan Z, Quan W. Laparoscopic Radical Gastrectomy for Resectable Advanced Gastric Cancer Within Enhanced Recovery Programs: A Prospective Randomized Controlled Trial. J Laparoendosc Adv Surg Tech A 2017; 27: 959-964 [PMID: 27875094 DOI: 10.1089/lap.2016.0057]

[20] Liu XX, Pan HF, Jiang ZW, Zhang S, Wang ZM, Chen P, et al. "Fast-track" and "Minimally invasive" surgery for gastric cancer. Chin Med J 2016;129:2294-300. doi: 10.4103/0366-6999.190659.

[21] Hou H, Ping X, Zhu Y, Zhao Z, Li Y, Li J. Dietary fiber alleviates intestinal barrier dysfunction in posttrauma rats. Clin Invest Med.2010;33(2):117-123.

[22] Yu G, Tang B, Yu PW, Peng ZH, Qian F, Sun G. Systemic and peritoneal inflammatory response after laparoscopic-assisted gastrectomy and the effect of inflammatory cytokines on adhesion of gastric cancer cells to peritoneal mesothelial cells. Surg Endosc. 2010;24(11):2860-2870.

[23] Svanfeldt M, Thorell A, Hausel J, Soop M, Rooyackers O, Nygren J, et al. Randomized clinical trial of the effect of preoperative oral carbohydrate treatment on postoperative whole-body protein and glucose kinetics. Br J Surg 2007;94:1342-50. doi: 10.1002/bjs.5919.

[24] Yermilov I, Jain S, Sekeris E, Bentrem DJ, Hines OJ, Reber HA, Ko CY, Tomlinson JS. Utilization of parenteral nutrition following pancreaticoduodenectomy: is routine jejunostomy tube placement warranted? Dig Dis Sci 2009; 54: 1582-1588 [PMID: 18958617 DOI: 10.1007/s10620-008-0526-1]

[25] Nanavati AJ. Fast Track Surgery in the Elderly: Avoid or Proceed with Caution? J Gastrointest Surg 2015; 19: 2292-2293 [PMID: 26394875 DOI: 10.1007/s11605-015-2946-5]

[26] Muehling BM, Ortlieb L, Oberhuber A, Orend KH. Fast track management reduces the systemic inflammatory response and organ failure following elective infrarenal aortic aneurysm repair. Interact Cardiovasc Thorac Surg 2011; 12: 784-788, doi: 10.1510/icvts.2010.262337. 
[27] Kehlet H. Multimodal approach to control postoperative pathophysiology and rehabilitation. $\mathrm{Br} \mathrm{J}$ Anaesth 1997; 78: 606-617 [PMID: 9175983]

[28] Mari G, Crippa J, Costanzi A, Mazzola M, Rossi M, Maggioni D. ERAS Protocol Reduces IL-6 Secretion in Colorectal Laparoscopic Surgery: Results From a Randomized Clinical Trial. Surg Laparosc Endosc Percutan Tech 2016; 26: 444-448 [PMID: 27783027 DOI: 10.1097/SLE.0000000000000324]

[29] Bona S, Molteni M, Rosati R, Elmore U, Bagnoli P, Monzani R, et al. Introducing an enhanced recovery after surgery program in colorectal surgery: a single center experience. World J Gastroenterol 2014; 20 : 17578-17587, doi: 10.3748/wjg.v20.i46.17578.

[30] Hoffmann H, Kettelhack C. Fast-track surgery--conditions and challenges in postsurgical treatment: a review of elements of translational research in enhanced recovery after surgery. Eur Surg Res 2012; 49: 24-34, doi: 10.1159/000339859.

[31] Du Clos TW. Function of C-reactive protein. Ann Med 2000; 32: 274-278.

[32] Pepys MB and Hirschfield GM. C-reactive protein: a critical update. J Clin Invest 2003; 111: 18051812.

[33] Giaccaglia V, Salvi PF, Cunsolo GV, et al. Procalcitonin, as an early biomarker of colorectal anastomotic leak, facilitates enhanced recovery after surgery. J Crit Care 2014; 29: 528-532.

[34] Miki Y, Toyokawa T, Kubo N, et al. C-reactive protein indicates early stage of postoperative infectious complications in patients following minimally invasive esophagectomy. World J Surg 2017; 41: 796-803.

[35] Asti E, Bonitta G, Melloni M, et al. Utility of C-reactive protein as predictive biomarker of anastomotic leak after minimally invasive esophagectomy. Langenbecks Arch Surg 2018; 403: 235-244.

[36] Muñoz JL, Ruiz-Tovar J, Miranda E, et al. C-reactive protein and procalcitonin as early markers of septic complications after laparoscopic sleeve gastrectomy in morbidly obese patients within an enhanced recovery after surgery program. J Am Coll Surg 2016; 222: 831-837.

[37] Garcia-Granero A, Frasson M, Flor-Lorente B, et al. Procalcitonin and C-reactive protein as early predictors of anastomotic leak in colorectal surgery: a prospective observational study. Dis Colon Rectum 2013; 56: 475-483.

[38] Benoit O, Faron M, Margot N, et al. C-reactiveprotein values after colorectal resection: can we discharge a patient with a C-reactive protein value $>100$ ? A retrospective cohort study. Dis Colon Rectum 2019; 62: 88-96.

[39] Hur H, Kim SG, Shim JH, et al. Effect of early oral feeding after gastric cancer surgery: a result of randomized clinical trial. Surgery.2011;149(4):561-568. 
[40] Kim JW, Kim WS, Cheong JH, Hyung WJ, Choi SH, Noh SH. Safety and efficacy of fast-track surgery in laparoscopic distal gastrectomy for gastric cancer: a randomized clinical trial. World J Surg. 2012;36(12):2879-2887.

[41] Feng F, Ji G, Li JP, et al. Fast-track surgery could improve postoperative recovery in radical total gastrectomy patients. World J Gastroenterol.2013;19(23):3642.

[42] Grantcharov TP, Kehlet $\mathrm{H}$. Laparoscopic gastric surgery in an enhanced recovery programme. $\mathrm{Br} \mathrm{J}$ Surg. 2010;97(10):1547-1551.

[43] Doig GS, Heighes PT, Simpson F, Sweetman EA, Davies AR. Early enteral nutrition, provided within 24 $\mathrm{h}$ of injury or intensive care unit admission, significantly reduces mortality in critically ill patients: a metaanalysis of randomised controlled trials. Intensive Care Med.2009;35(12):2018-2027.

[44] Park WY, Thompson JS, Lee KK. Effect of epidural anesthesia and analgesia on perioperative outcome: a randomized, controlled Veterans Affairs cooperative study. Ann Surg. 2001;234(4):560.

[45] Nelson R, Tse B, Edwards S. Systematic review of prophylactic nasogastric decompression after abdominal operations. Br J Surg.2005;92(6):673-680.

\section{Supplementary Files}

This is a list of supplementary files associated with this preprint. Click to download.

- Enhancedrecoveryaftersurgery02Supplementarytable1.pdf 